\title{
IoT-Based Fire Safety System Using MQTT Communication Protocol
}

\author{
Yuen Chark See ${ }^{1^{*}}$, En Xiang Ho ${ }^{2}$ \\ ${ }^{1}$ Universiti Tunku Abdul Rahman, \\ Lee Kong Chian Faculty of Engineering \& Science, Sungai Long Campus, Jalan Sungai Long, Bandar Sungai Long, \\ 43200 Kajang, Selangor, MALAYSIA
}

*Corresponding Author

DOI: https://doi.org/10.30880/ijie.2020.12.06.024

Received 22 February 2020; Accepted 19 July 2020; Available online 30 July 2020

\begin{abstract}
Fire can be made useful for various purposes. However, uncontrollable fire may result in property damage and human death. The major factor of fire deaths is due to excessive smoke inhalation. Therefore, early detection of fire is crucial in fire detection systems. The conventional fire detection system does not come with a false alarm prevention system. Besides, the system is unable to tell the exact location of the fire. In this project, an Internet of Things (IoT) based fire safety system is developed to overcome these problems. The proposed system consists of three major parts which are the detector, processing unit and surveillance. The detector unit is an integration of ESP32, carbon monoxide sensor, ionization smoke detector, buzzer, temperature and humidity sensor. As the processing unit, Raspberry Pi is used to run the Node-RED application, which processes the data and performs monitoring. The communication between the detector and processing unit is based on the Message Queuing Telemetry Transport (MQTT) protocol. A surveillance unit is where a camera is installed to monitor the condition of the surrounding. The response of the system is based upon the sensor's values or the user's response. Once the fire breakout is confirmed, the system will immediately sound the alarm, and Global Positioning System (GPS) coordinates and floor plan of the accommodation will send to the nearby fire station. The floor plan is developed to track the exact location of the fire. Experiments are carried out on the proposed fire safety system, and encouraging results are produced.
\end{abstract}

Keywords: ESP32, Fire Safety System, Internet of Things, MQTT, Node-RED

\section{Introduction}

Fire was discovered by humankind many years ago. Until today, it is still important to human in their daily livings. Fire is useful when it is under control. However, it may lead to destruction if it is uncontrolled. Fires can be classified into two groups - flaming fire and smoldering fire. Flaming fire produces a large volume of flames but smaller volume of smoke. Usually, flaming fire is due to the ignition of materials such as flammable materials or liquids. A smoldering fire is a type of fire that is slow in spreading, low in temperature, and flameless. It is a condition in which the material is being burned slowly with smoke but without flame. The smoke produced by such fire may contain toxic chemicals such as cyanide and carbon monoxide.

Fig. 1 shows the total number of building fires from 2012-2016 in Malaysia [1]. It can be observed that the number of reported fire cases over the five years remains high at the average of five thousand cases. The types of buildings can be further broken down into a few categories, such as residential, retail, factory, institution, office, public place, and others. Among these categories, residential premises contributed to the highest building fire incident each year. Referring to the National Fire Protection Association (NFPA) [2], most fire casualties are due to the inhalation of smoke rather than burns. Even before the victims can reach the exit of the building, they were already intoxicated by the smoke itself. One of the reasons is that most of the oxygen was consumed by the fire itself during burning, which 
causes the oxygen level in the air to decrease. Table I shows the symptoms when the oxygen level in the air decreases. Smoke inhalation is the major factor for most of the fire deaths rather than burns. If smoke can be detected earlier, the victim will be able to have enough time to escape the building. Therefore, it is important to install a fire safety system with smoke detectors which can provide early detection of fire and alert the victims.

The conventional fire alarm system consists of various smoke detectors or calls points, which is being connected to the control panel using wires. All the components and devices in the system are controlled or monitored by the fire alarm control panel (FACP) based on various zones. One of the disadvantages of the current fire detection system is not able to provide the exact location of the fire [3]. It only provides the information on which zone is on fire. Besides, the conventional fire detection system was not equipped with a false alarm prevention system [4]. False alarm of the system could be triggered by high humidity, dust or insects, chemical odors or cigarette smoke

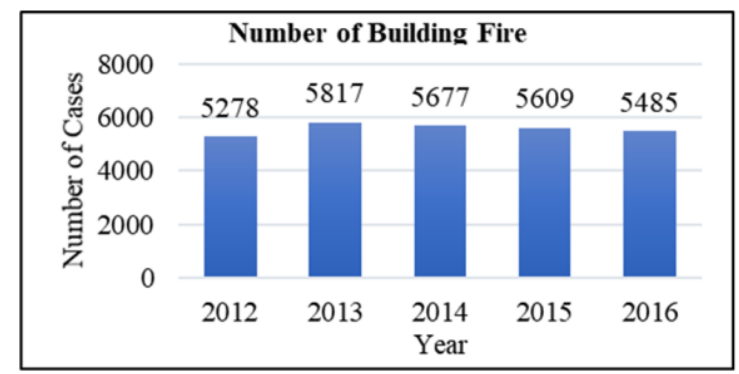

Fig. 1 - Malaysia Building Fire Statistics [1]

Table 1 - Oxygen levels and effects [2]

\begin{tabular}{cl}
\hline Oxygen Levels at (\%) & \multicolumn{1}{c}{ A person experience } \\
\hline 21 & Normal outside air \\
17 & Impaired judgment and coordination \\
12 & Headache, dizziness, nausea, fatigue \\
9 & Unconsciousness \\
6 & Respiratory arrest, cardiac arrest, death \\
\hline
\end{tabular}

The novel representation of this paper is to develop an IoT fire safety system utilizing MQTT to establish communication between devices. The proposed system can store data such as temperature, humidity and carbon monoxide concentration on a cloud storage system. Additionally, the system will notify the authority whereabout of the fire and able to detect false fire alarm.

\section{Related Work}

The two most common smoke detectors used are photoelectric and ionization smoke detectors. Ionization smoke detectors are good at detecting flaming fires, while photoelectric smoke detectors are better at detecting smoldering fires.

The ionization smoke detector mainly consists of a detector chamber and a small amount of radioactive element called Americium-241, with a half-life of 432 years [5]. Such a radioactive material will emit radiation consisted of alpha particles. Under normal conditions, electricity cannot flow through the air because air is considered as an insulator. However, when the alpha particles are produced, they will collide with the air particles inside the ionization chamber. Such a collision will cause the air particles to be ionized thus, producing ions and electrons. When the smoke gets inside the chamber, it will interfere with the flow of the alpha particles. Fewer ions and electrons will be produced and therefore interrupts the current flow. When the current drops to a certain level, it will trigger the alarm. In the absence of smoke, the number of current flows will return to normal, and the alarm stops. This type of smoke detector will be able to sense the small, invisible particles, and therefore, it provides a better response toward a flaming fire [6, 7].

The photoelectric smoke detector consists of a Light Emitting Diode (LED) and a photosensor inside the chamber $[7,8]$. The function of the photosensor is to detect the presence of light. The purpose of LED is to generate a beam of light across the chamber, but it is not directed towards the photosensor. When smoke enters the chamber, it will cause the light beam to be interrupted and scattered. Under this condition, some of the light might be scattered to the photosensor unintentionally. When the sensor detected a certain amount of light, it will trigger the alarm. The photoelectric smoke detector is better in detecting a slow-developing fire, which produces more smoke than flames.

A dedicated fire alert detection system was developed using a Zigbee wireless network [9]. The temperature sensor is used for fire detection. When the sensor detects heat, it will send a signal to the Arduino Uno. The buzzer will be turned on when the temperature reached a pre-set value. The information will be sent to the laptop for monitoring via a 
ZigBee wireless network using the Xbee transmitter and Xbee receiver. However, one of the disadvantages is that the signal is confined to 10 meters. Another fire detection system was also developed by Islam et al. [10] using a similar wireless network. This system has a localizing capability. The authors used three sensors for the localization of a fire, adapting the Trilateration technique. All the data obtained will be collected by the ZigBee network coordinator and sent to the Wi-Fi network using Arduino and Arduino Ethernet Shield. However, the authors claimed that the system would potentially require a relatively high initial cost.

Instead of using sensors, a system that utilized camera image processing to detect a fire outbreak was implemented [11]. This is done by comparing the original image under the normal condition without fire and the current image captured. Fire can be detected based on different classification such as flame type, smoke type, and sparkling type. Kong et al. [12] developed a fire flame detection using a surveillance video camera. This system was implemented using logistic regression and temporal smoothing. Characteristics of the fire, such as size, motion, and color components of the fire flame, will be taken into consideration. Temporal smoothing is used to minimize the rate of getting a false alarm. Another method for early fire detection based on video processing is by using the smoke-pixel judgment, which consists of two decision rules [13]. The first rule is the chromaticity-based static decision rule. The second rule is the diffusion-based dynamic characteristic decision rule. The static decision rule is determined based on the greyish color of the smoke during the burning process. For dynamic decision rule, the spreading characteristics of the smoke are taken into consideration.

Imteaj et al. [14] proposed an Internet of Things (IoT) based fire alarming system. The system was able to detect fire as well as providing the location where the fire occurred. Raspberry Pi 3 is used as the main controller to control the Arduino, which is integrated with sensors and camera. When a fire occurred, the system will send a message which includes the image captured and the Arduino's location to the admin. The admin is required to confirm the fire outbreak. Once confirmed, the alarm will be raised, and a message will be sent to the nearby fire brigade. In line with the IoT based fire alarm system, W.h.Dong et al. [15] developed a wireless communication protocol that has the capability of rapid-fire detection. The protocol operates on low power consumption utilizing the 433M radio frequency architecture. Karwan et al. [16] proposed a Wireless Sensor Network (WSN) method in designing a fire alarm system. The authors claimed that with the WSN method, the cost of the fire alarm system would be reduced. This system was utilizing the Arduino devices and different types of sensors to obtain temperature, humidity, gas, and flame level data for analysis. Besides, a fire monitoring and controlling system were developed by making use of various sensors along with Global System for Mobile Communications (GSM), which is used to inform the user through mobile messages [17]. Besides, it also utilized the Global Positioning System (GPS) technology to locate the place where the fire occurs. When a fire occurred, the sensors will send a signal to the microcontroller ARM LPC2148. Then, the microcontroller will activate the emergency alarm as well as the water sprinklers for on-site control of fire. At the same time, the location of the incident will be obtained using the GPS module, and a Short Message Service (SMS) will be sent to the fire station.

\section{Methodology}

The flowchart of the proposed fire alarm system is shown in Fig. 2. Based on the flowchart, when the ionization smoke detector is triggered, or the carbon monoxide (CO) concentration is above or equal to 35 parts per million (ppm), an email will be sent to notify the user. However, when the detector is triggered and the concentration of $\mathrm{CO}$ is above or equal to $35 \mathrm{ppm}$ or $100 \mathrm{ppm}$, the alarm will be raised automatically. Apart from the email and message notification, an immediate voice call will be sent to the user through the internet. The chosen $\mathrm{CO}$ concentration values are based on the research paper by Goldstein [18].

According to the Malaysian Meteorological Department, the highest temperature recorded in 2017 is $37.6{ }^{\circ} \mathrm{C}$ in Mulu, Sarawak [19]; the highest recorded temperature in a room in Kuala Lumpur was $32.6^{\circ} \mathrm{C}$ [20]. The sensor used in the proposed system has a temperature accuracy of $\pm 2{ }^{\circ} \mathrm{C}$, with the threshold value selected at $37{ }^{\circ} \mathrm{C}$. However, the threshold value for the temperature can be adjusted according to the condition of the environment. Referring to the flowchart in Fig. 2, the alarm will be raised if the temperature is above $37^{\circ} \mathrm{C}$ and when the temperature is higher than $49^{\circ} \mathrm{C}$, the GPS coordinates of the building will be sent to the nearest fire station.

The proposed system consists of three major parts, namely the processing unit, detector, and surveillance. The block diagram of the proposed system is shown in Fig. 3 and will be discussed in the following section.

\subsection{Processing Unit}

The primary processing unit used in this system is Raspberry Pi 3 Model B+ [21] with Hass.io operating system (OS) [22] running on it. Node-RED (a programming tool for IoT) [23] was installed to communicate with all the devices in the system. 


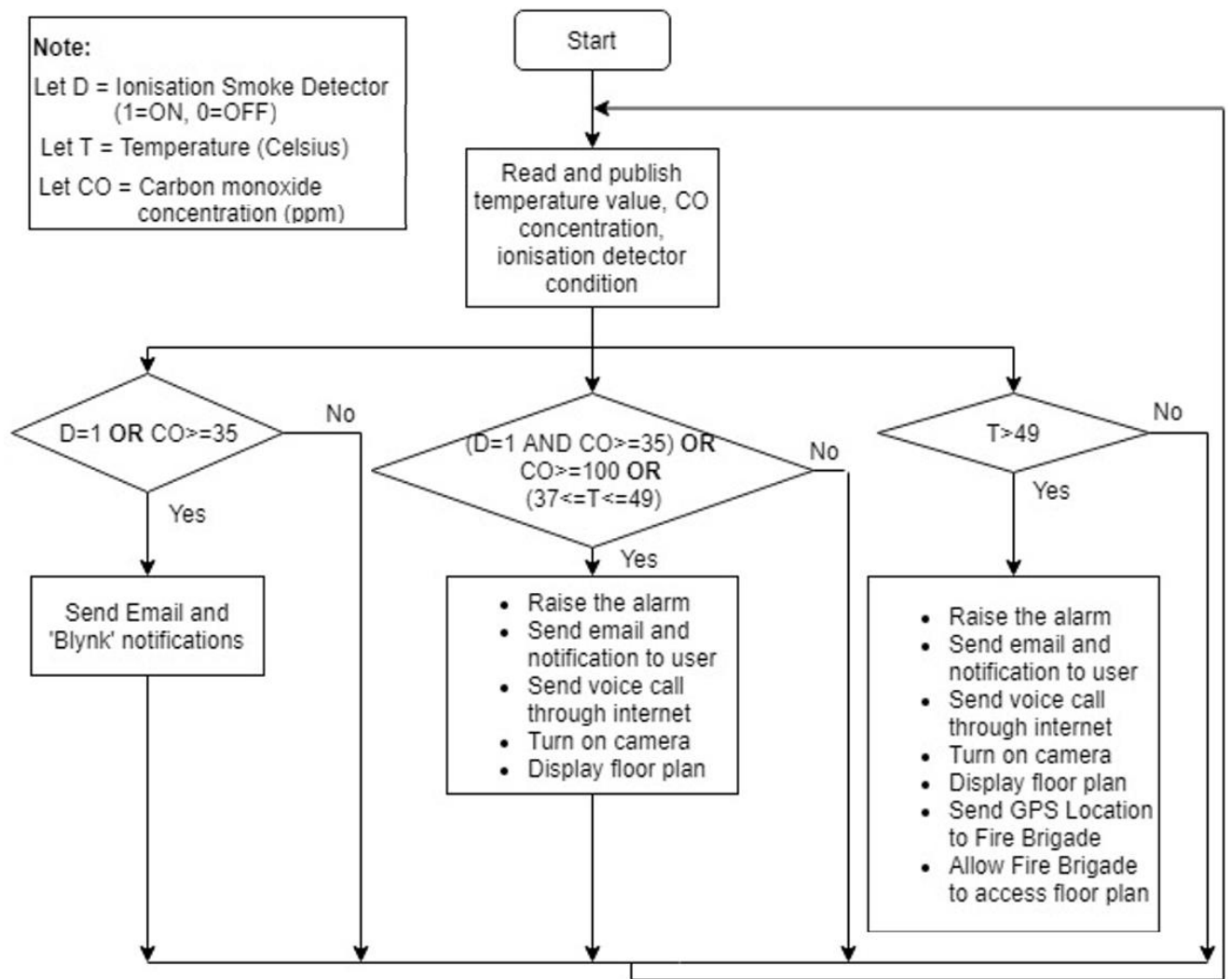

Fig. 2 - Flowchart of the proposed system

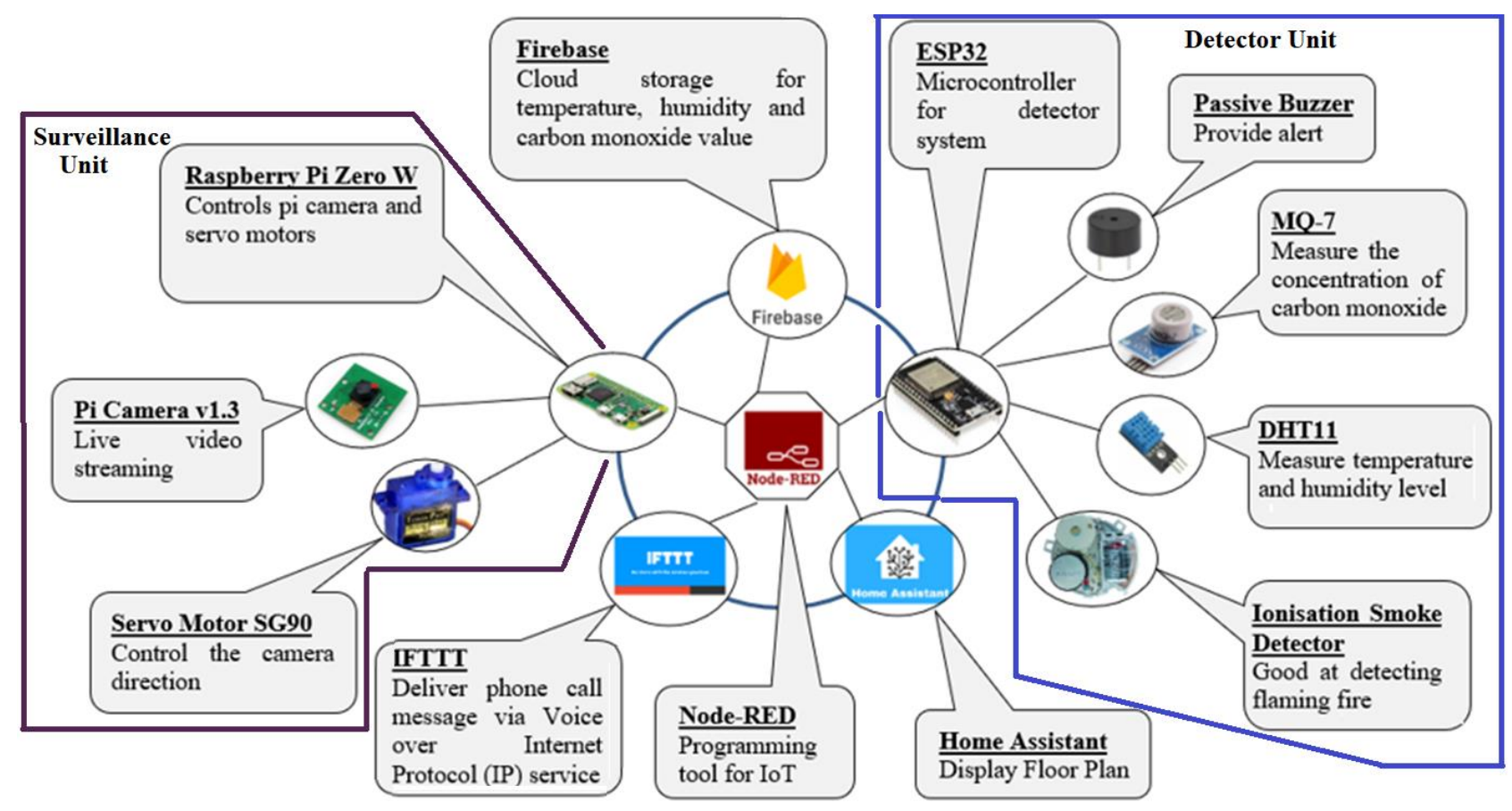

Fig. 3 - Proposed Fire Alarm System

\subsection{Detector Unit}

Detector unit is an integration of ESP32, DHT11, MQ-7, buzzer, and ionization smoke detector. MQ-7 is used to measure the concentration of Carbon monoxide $(\mathrm{CO})$ in the air, while DHT11 is used to measure the temperature and humidity levels of the surrounding. The ionization smoke detector consists of 1.0 microcuries of Americium-241. It is 
powered up with a $9 \mathrm{~V}$ battery. The ionization smoke detector is good in detecting flaming fire. ESP32 is the microcontroller for the detector unit. It is responsible for obtaining the data from the sensors and the triggering of the buzzer.

\subsection{Surveillance Unit}

The surveillance unit is to enable the user to observe the condition of the surrounding through live video streaming and, therefore, to identify whether it is a real fire. It consists of Raspberry Pi Zero W, Servo Motor SG90, and Raspberry Pi Camera v1.3. The Raspberry Pi Zero W is an improved version of the Pi Zero family, which comes with added connectivity, consisting of wireless local area network (LAN), Bluetooth 4.1, and Bluetooth Low Energy (BLE). In the proposed system, it will be used to control the Raspberry Pi Camera v1.3 and the SG90 servo motors. The SG90 servo motor is used to control the direction of the camera.

\subsection{MQTT as the Communication Protocol}

The data collected from the sensors will be sent to the processing unit by the ESP32 via Message Queuing Telemetry Transport (MQTT) communication protocol [24]. MQTT is a lightweight publish or subscribing messaging transport protocol for machine-to-machine communications that runs on top of the Transmission Control Protocol (TCP) stack [25-26]. The security of the MQTT message can be further enhanced by using TTL/SSL internet security encryption. Fig. 4 shows the communication model of the system between ESP32 and Node-RED. Mosquitto broker will be installed on the Raspberry Pi to manage the information exchange among the clients. A broker is a software that functions to receive all the information and messages from the publishing clients. It filters the messages and sends the information to the subscribing clients. MQTT is adapting a central broker where it can reduce the amount of packets to be sent to the internet and reduces the memory usage requires by the clients. The red-colored wordings represent the MQTT topics. ESP32 will obtain the values from the sensors and publish them on the topics subscribed by Node-RED. The information received will be updated and displayed on the Node-RED user interface regularly. When two or more sensors input are above the typical values, Node-RED will publish the message "on" to the topic "esp32/alarm" to inform the ESP32 to trigger the alarm.

\subsection{Node-RED}

Node-RED [23] is a visual tool for providing the connections of different devices for the Internet of Things (IoT). It was an open-source flow-based development tool. In this system, it is used to communicate with all the devices in the fire safety system.

\subsection{IFTTT}

If This, Then That (IFTTT) is a free web-based service used to create chains of simple conditional statements that enable various apps and devices to work together [27]. Applets help to bring various services together to perform a task. Fig. 9 (b) shows an applet that was created by utilizing both "Webhooks" and "VoIP Calls" services. With the VoIP Calls service, phone call messages will be delivered to the user when any fire or excessive smoke is detected.

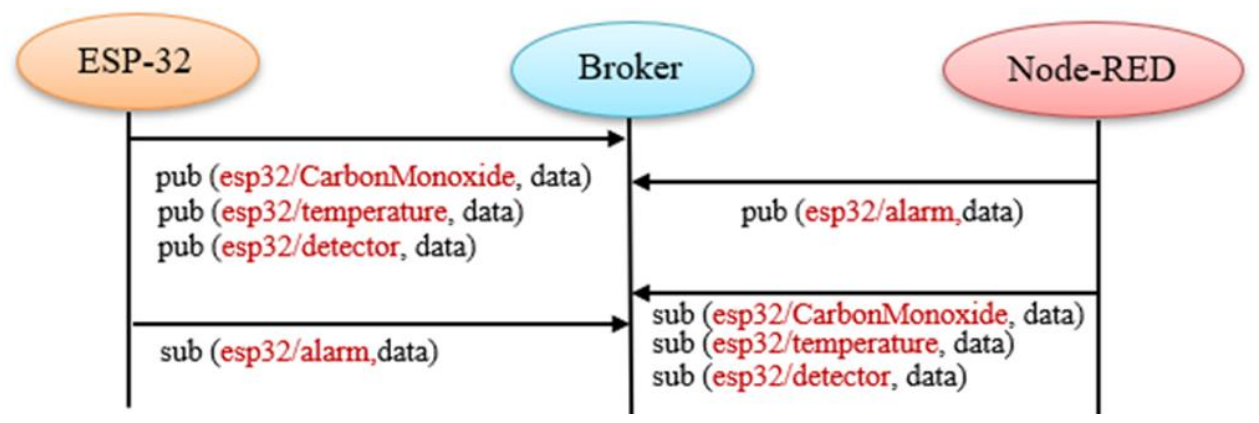

Fig. 4 - Proposed MQTT communication model

\subsection{Home Assistant}

Home Assistant (HA) [22] is an open-source platform that allows the user to track, control and automate different types of smart devices. In this project, Hass.io is used as the operating system (OS) and install in Raspberry Pi 3 Model $\mathrm{B}+$. The installation of Hass.io turns Raspberry Pi into an automation hub. 


\subsection{Firebase Realtime Database}

The readings obtained from the sensors will be stored in the Firebase Realtime database [28]. The data stored can be retrieved and displayed on the user interface in a graphical form. Fig. 5 (a) shows the output values of the sensors that are stored in the Firebase Realtime Database. The data is categorized into three types which are the humidity level, carbon monoxide concentration, and the temperature level. The data format stored in the Firebase database is shown in Fig. 5 (b). The first number " 214 " represents the sensor output value while the second number "1553..." represents time in JavaScript Object Notation (JSON) format.
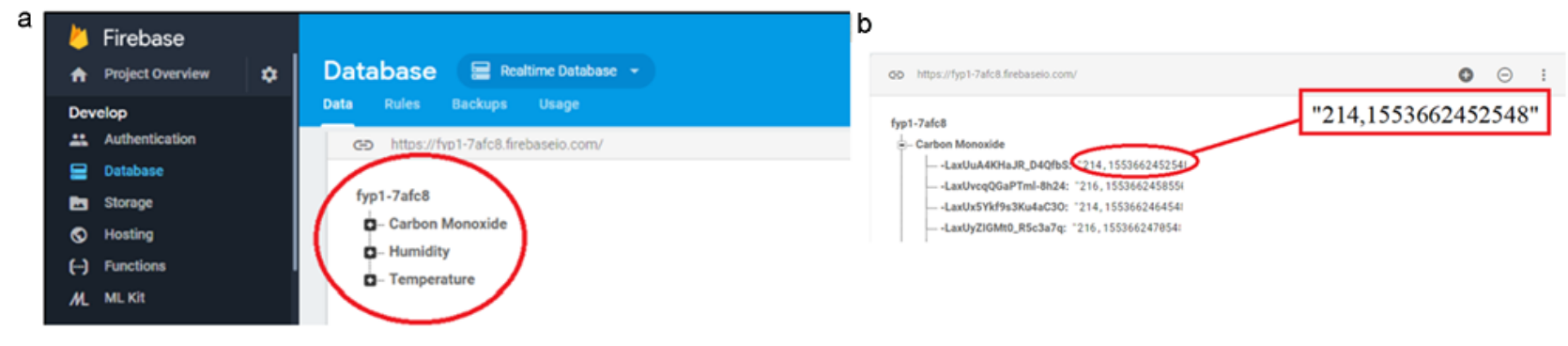

Fig. 5 - (a) Data type by category; (b) Data format

\section{Results and Discussions}

Fig. 6 (a) shows the Raspberry Pi 3 Model B+, which is used as the main processing unit for the proposed system. The Universal Serial Bus (USB) flash drive is used to configure the Wi-Fi connection. Fig. 6 (b) is the surveillance unit used to monitor the condition of the surrounding. Fig. 6 (c) shows the hardware prototype of the detector unit. The detector unit consists of ESP32, MQ-7, DHT11, and buzzer. The ESP32 will obtain the data from the sensors and send them to the processing unit via the MQTT communication protocol. Two experiments were conducted to test the proposed system. These experiments will be discussed in the following sections.
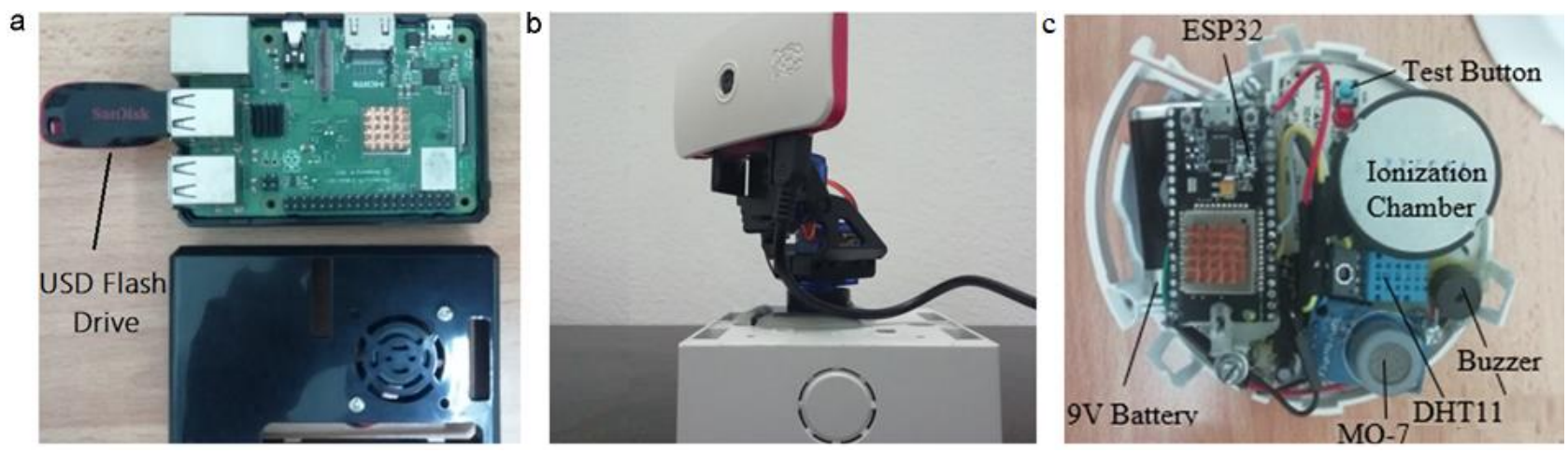

Fig. 6 - (a) Processing unit; (b) Surveillance unit; (c) Detector unit (inside view)

\subsection{Experiment 1: Verification of System Response with Burning Candle}

A few chambers are built to simulate multiple sensors in multiple locations in the same building. One of the chambers is shown in Fig. 7. Tea candle is used to simulate a flaming fire (Fig 7 (a)), and incense burning is used to simulate a burning smoke (Fig. 7 (b)). Fig. 7 (a) shows the conducted experiment to test the functionality of the system under flaming fire. The burning candle is used to represent a flaming fire that does not produce much smoke. The data obtained from the sensors are recorded in Table 2. It is observed that the temperature increased significantly within the first 120 seconds. The reading of carbon monoxide is also increased accordingly. Fig. 8 (a) shows the user interface created using Node-RED. The status of the ionization smoke detector, alarm, and the real-time outputs from the sensors are displayed on the dashboard.

Multiple detectors were installed at their respective location according to the floor plan shown in Fig. 8 (b). The circular icons are used to represent the status of the ionization smoke detector. A green icon indicates the detectors are in the normal condition. When the detector is triggered, it changes to red. There are two fire warnings, as indicated in Fig. 8 (b). The floor plan would be very useful in searching for the origin of fire during firefighting operation as well as the evacuation process in the high-rise buildings. In the meantime, voice message and email will be sent to the user or related party to inform the activation of the alarm. Email and voice interaction are shown in Fig. 9 (a) and (b), respectively. The Global Positioning System (GPS) will identify the coordinates of the building and contact the nearby 
fire brigade station. The fire-brigade department will be able to access the related information such as the coordinate of the incident, the floor plan of where the fire alarm is triggered, and the control of the surveillance camera. Fig. 9 (c) shows the map where the fire alarm is activated. By clicking the red oval spot, the house plan, as in Fig. 8 (b) will appear together with real-time surveillance.
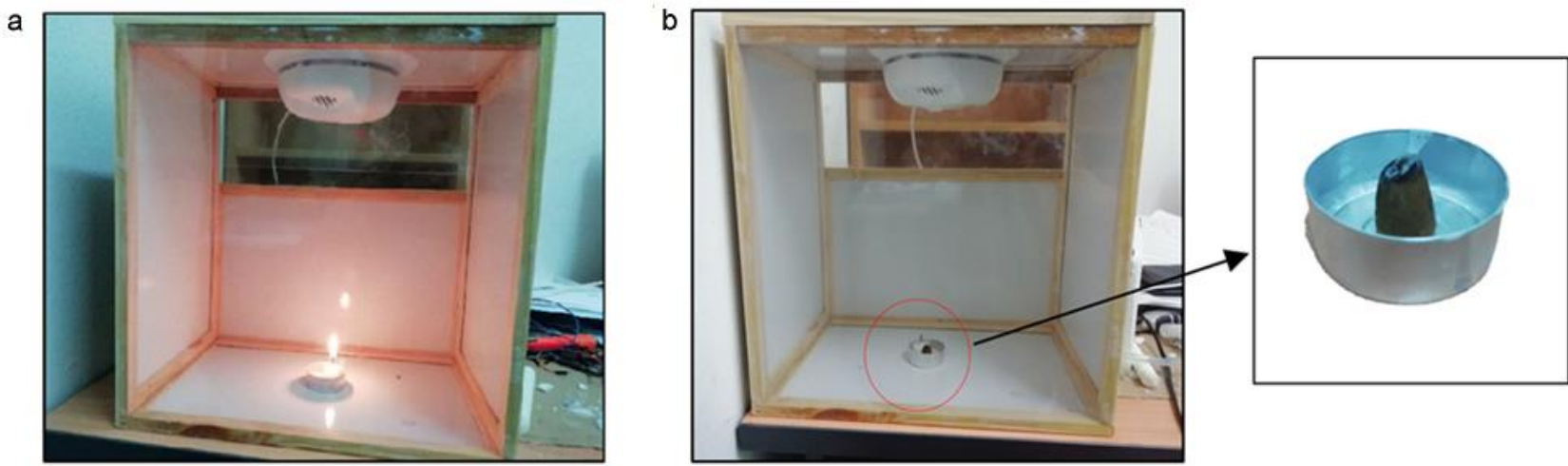

Fig. 7 - (a) Experiment with a burning candle; (b) Experiment with burning incense

Table 2 - Sensors Reading (Experiment 1)

\begin{tabular}{cccc}
\hline Time (s) & Temperature $\left({ }^{\circ} \mathbf{C}\right)$ & Humidity $(\boldsymbol{\%})$ & $\begin{array}{c}\text { Carbon } \\
\text { Monoxide }(\mathbf{p p m})\end{array}$ \\
\hline 10 & 33 & 60 & 212 \\
30 & 34 & 64 & 360 \\
60 & 35 & 69 & 528 \\
90 & 36 & 70 & 693 \\
120 & 41 & 70 & 720 \\
\hline
\end{tabular}
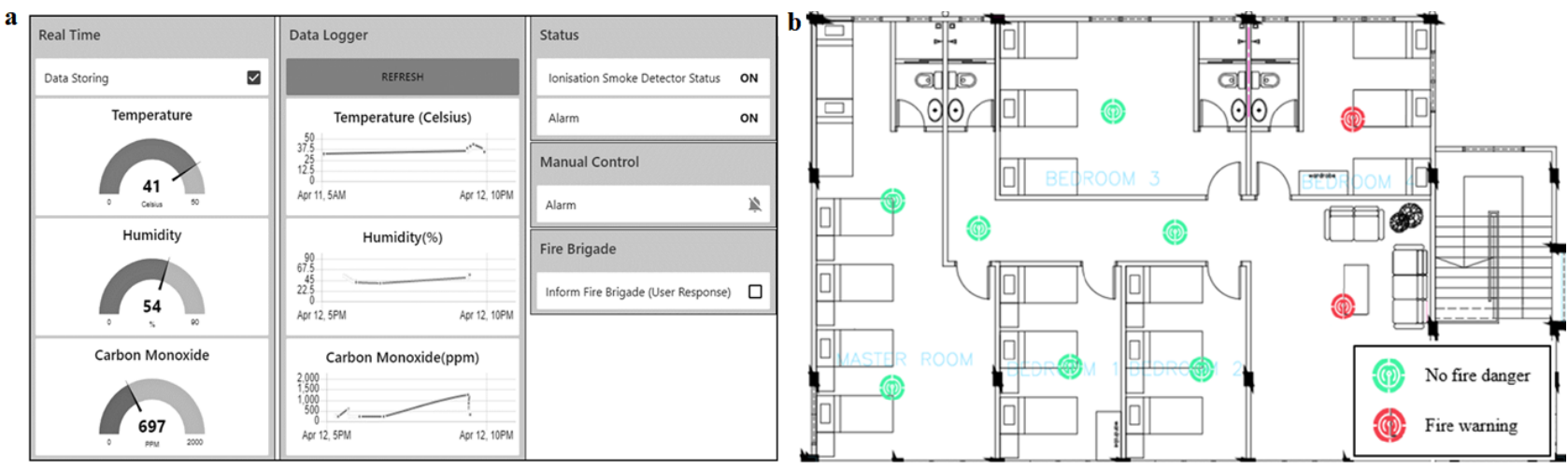

Fig. 8 - (a) Node-RED dashboard - Smoke detector; (b) Floor plan showing the sensors location

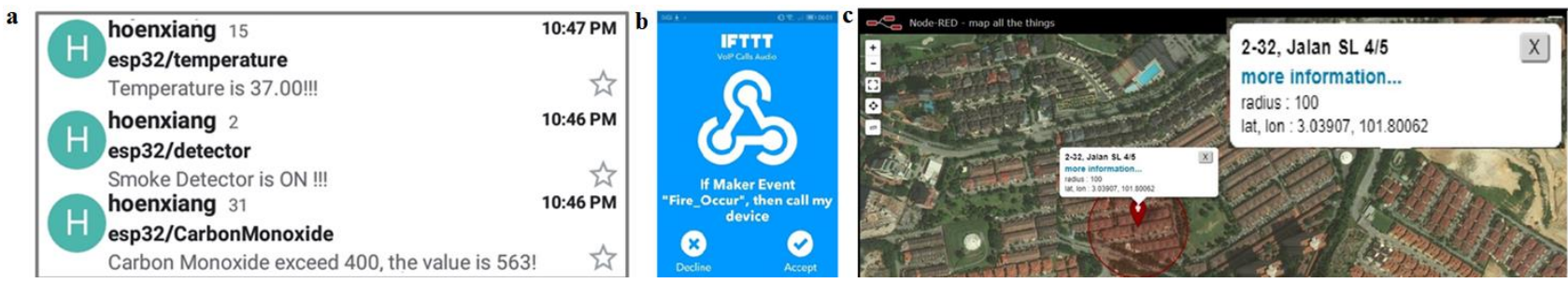

Fig. 9 - (a) Email interaction; (b) Voice interaction using IFTTT; (c) Map indicating the location of fire 


\subsection{Experiment 2: Verification of System Response with Burning Incense}

This experiment is conducted to test the functionality of the system under a smoldering fire. Burning incense is used as it only produces smoke. The setup of the experiment is shown in Fig.7 (b). Data obtained from the sensors are recorded in Table 3. The result shows that the temperature remains the same within 60 seconds. However, there is a significant increase in the concentration of carbon monoxide in the air as compared to the previous result, as in Table 1 (Experiment 1). When the threshold level of carbon monoxide concentration is reached, voice message and email will be sent to the user as in Fig. 9. Besides, the circular icon on the floor plan also changed from green to red when the detector is triggered, as in Fig. 8(b).

Table 3: Sensors Reading (Experiment 2)

\begin{tabular}{cccc}
\hline Time $(\mathrm{s})$ & $\begin{array}{c}\text { Temperature } \\
\left({ }^{\circ} \mathrm{C}\right)\end{array}$ & $\begin{array}{c}\text { Humidity } \\
(\%)\end{array}$ & $\begin{array}{c}\text { Carbon } \\
\text { Monoxide } \\
(\mathrm{ppm})\end{array}$ \\
\hline 10 & 34 & 59 & 256 \\
20 & 34 & 59 & 626 \\
40 & 34 & 59 & 1245 \\
60 & 34 & 61 & 1261 \\
\hline
\end{tabular}

\section{Conclusions and Recommendations}

The primary purpose of this research is to develop an IoT based Fire Safety System. The proposed system can offer solutions to the problem related to the conventional system. To reduce false alarms, multiple sensors are used instead of one. Besides, the user able to monitor the condition of the surrounding through the webcam. The fire source can be located with the help of the floor plan. This information would be very useful during the firefighting operation as well as the evacuation process. When the fire occurred, the user will be notified through email and voice message. At the same time, the location of the building will be sent to the nearby fire brigade. An experiment was conducted by simulating a flame using a tea candle to prove the functionality of the system. In the future, the network of the system can be widened by including water sprinkler and main power supply control Besides, strobe lights could be integrated into the smoke detector system for individuals with hearing problems.

\section{Acknowledgement}

This research was supported by University Tunku Abdul Rahman FYP research fund.

\section{References}

[1] Fire and Rescue Department of Malaysia. (2016). Laporan tahunan JBPM. http://www.bomba.gov.my/index.php/pages/view/746? mid= 251

[2] National Fire Protection Association (2018). Reporter's Guide: The consequences of fire. https://www.nfpa.org/News-and-Research/Publications-and-media/Press-Room/Reporters-Guide-to-Fire-andNFPA/Consequences-of-fire\#b/

[3] Vijayalakshmi, S. \& Muruganand ,S. (2018). Fire alarm based on spatial temporal analysis of fire in video. 2nd International Conference on Inventive Systems and Control (ICISC)

[4] Hasan, M. M. \& Razzak, M. A (2016). An automatic fire detection and warning system under home video surveillance. Signal Processing \& Its Applications (CSPA), 2016 IEEE 12th International Colloquium (pp. 258262)

[5] Brain, M. (2000). How smoke detectors work. https://home.howstuffworks.com/home-improvement/householdsafety/smoke1.htm

[6] Lipu, M. S. H., Karim, T. F., Rahman, M. L., \& Sultana, F. (2010). Wireless security control system \& sensor network for smoke \& fire detection. 2010 IEEE International Conference on Advanced Management Science .

[7] Serrano, J. E. M. N. (2017). Study, design and validation of a framework model for smoke and particle-filled atmospheres. Universidad Politécnica de Madrid

[8] Ma, J., Yang, X., \& Wang, P. (2017). Design of the intelligent smoke alarm system based on photoelectric smoke. Second International Conference on Photonics and Optical Engineering

[9] Fuzi, M. F. M., Ibrahim, A. F., Ismail, M. H., \& Ab Halim, N. S. (2014). HOME FADS: A dedicated fire alert detection system using ZigBee wireless network. IEEE 5th Control and System Graduate Research Colloquium

[10] Islam, T., Rahman, H. A., \& Syrus, M. A. (2015). Fire detection system with indoor localization using ZigBee based wireless sensor network. International Conference on Informatics, Electronics \& Vision (ICIEV)

[11] Kwon, O.-H., Cho, S.-M., \& Hwang, S.-M. (2008). Design and implementation of fire detection system. Advanced Software Engineering and Its Applications 
[12] Kong, S. G., Jin, D., Li, S., \& Kim, H. (2016). Fast fire flame detection in surveillance video using logistic regression and temporal smoothing. Fire Safety Journal, 79, 37-43

[13] Chen, T.-H., Yin, Y.-H., Huang, S.-F., \& Ye, Y.-T. (2006). The smoke detection for early fire-alarming system base on video processing. International Conference on Intelligent Information Hiding and Multimedia

[14] Imteaj, A., Rahman, T., Hossain, M. K., Alam, M. S., \& Rahat, S. A. (2017). An IoT based fire alarming and authentication system for workhouse using Raspberry Pi 3. International conference on electrical, computer and communication engineering (ECCE)

[15] Dong, W.-h., Wang, L., Yu, G.-Z., \& Mei, Z.-b. (2016). Design of wireless automatic fire alarm system. Procedia Engineering, 135, 413-417

[16] Muheden, K., Erdem, E., \& Vançin, S. (2016). Design and implementation of the mobile fire alarm system using wireless sensor networks. IEEE 17th International Symposium on Computational Intelligence and Informatics

[17] Gaikwad, K., Quadri, A., Akshada, S., \& Reshma, Z. (2016). Fire monitoring and control system. Proceedings of International Research Journal of Engineering and Technology (IRJET)

[18] Goldstein, M. (2008). Carbon monoxide poisoning. Journal of Emergency Nursing, 34(6), 538-542

[19] Malaysian Meteorological Department (2017). Cimate http://www.met.gov.my/content/pdf/penerbitan/laporantahunan/laporantahunan2017.pdf

[20] Jamaludin, N., Mohammed, N. I., Khamidi, M. F., \& Wahab, S. N. A. (2015). Thermal comfort of residential building in Malaysia at different micro-climates. Procedia-Social and Behavioral Sciences, 170, 613-623

[21] The Raspberry Pi 3 Model B+ overview. (2018). https://static.raspberrypi.org/files/product-briefs/Raspberry-PiModel-Bplus-Product-Brief.pdf

[22] Technical documentation of Home Assistant. (2019). https://home-assistant.io/hassio/.

[23] Node-RED guide. (2019). http://noderedguide.com/

[24] Technical documentation for MQTT. (2019). http://mqtt.org/documentation

[25] Karagiannis, V., Chatzimisios, P., Vazquez-Gallego, F., \& Alonso-Zarate, J. (2015). A survey on application layer protocols for the internet of things. Transaction on IoT and Cloud computing, 3(1), 11-17

[26] Yassein, M. B., \& Shatnawi, M. Q. (2016). Application layer protocols for the Internet of Things: A survey. International Conference on Engineering \& MIS (ICEMIS)

[27] Ovadia, S. (2014). Automate the internet with "if this then that"(IFTTT). Behavioral \& social sciences librarian, 33(4), 208-211

[28] Moroney, L. (2017). The firebase realtime database. In The Definitive Guide to Firebase (pp. 51-71): Springer 